\title{
An Outbreak Investigation of Burkholderia Cepacia Infections Related With Contaminated Chlorhexidine Mouthwash Solution in a Tertiary Care Center in Turkey.
}

Huseyin Bilgin ( $\nabla$ huseyin.bilgin@marmara.edu.tr)

Marmara Universitesi Egitim ve Arastirma Hastanesi https://orcid.org/0000-0002-5048-9970

Gülşen Altınkanat Gelmez

Marmara Universitesi Egitim ve Arastirma Hastanesi

Fatma Bayrakdar

Public Health Institute of Turkey

Elvan Sayın

Marmara Universitesi Egitim ve Arastirma Hastanesi

Fethi Gül

Marmara Universitesi Egitim ve Arastirma Hastanesi

Nazlı Pazar

Marmara Universitesi Egitim ve Arastirma Hastanesi

Gulcan Culha

Marmara Universitesi Egitim ve Arastirma Hastanesi

Serap Suzuk Yildiz

Public Health Institute of Turkey

İsmail Cinel

Marmara Universitesi Egitim ve Arastirma Hastanesi

Volkan Korten

Marmara Universitesi Egitim ve Arastirma Hastanesi

Research

Keywords: Burkholderia cepacia, outbreak, intensive care unit, chlorhexidine mouthwash, ventilatorassociated-pneumoniae, Healthcare associated infection, contamination, outbreak investigation

Posted Date: January 26th, 2021

DOI: https://doi.org/10.21203/rs.3.rs-153265/v1 
License: (c) (i) This work is licensed under a Creative Commons Attribution 4.0 International License. Read Full License 


\section{Abstract}

Background: We report a nosocomial outbreak caused by Burkholderia cepacia that occurred among six patients admitted in the medical and surgical intensive care unit between 04 March 2019 and 02 April 2019 in Istanbul, Turkey.

Methods: The outbreak investigation was launched on 11 March 2019 five days after the detection of $B$. cepacia in four different patients. We defined potential reservoirs and started environmental screening. We sampled the liquid solutions used in patient care activities. Pulse-field gel electrophoresis (PFGE) was performed to determine the genetic relatedness of environmental and patient samples.

Results: $B$. cepacia was isolated in tracheal aspiration cultures of six patients. Three out of six patients developed healthcare-associated pneumoniae due to B. cepacia. Environmental cultures in the ICUs revealed $B$. cepacia growth in $2 \%$ chlorhexidine-gluconate mouthwash solution that been used in the colonized patients as well as in samples obtained from the unused products. PFGE revealed the patient and a specific batch of chlorhexidine mouthwash solution samples had a $96 \%$ similarity.

Conclusion: Contamination of medical solutions used in critical patient care could cause outbreaks and should be detected early by infection control teams.

\section{Introduction}

Burkholderia cepacia is an aerobic, gram-negative, non-fermentative bacteria frequently isolated from water and solutions [1]. It can survive in fluids, antiseptic solutions, and the healthcare environment for long durations. Burkholderia cepacia complex is significant in cystic fibrosis patients, patients with structural lung diseases, and immunocompromised patients [2]. A variety of sources causes B. cepacia outbreaks.

Previous reports have demonstrated transmission from contaminated liquids or moist environmental surfaces in hospital settings [3-5]. Here we describe a nosocomial outbreak caused by Burkholderia cepacia between 04 March and 02 April 2019 in a tertiary care center in Istanbul, Turkey. The outbreak occurred in patients admitted in intensive care units (ICU) with and without structural lung disease. We report the identification of the source as contaminated $2 \%$ chlorhexidine $(\mathrm{CHG})$ mouthwash solution.

\section{Methods}

\section{Setting:}

The hospital is a tertiary care center with 659 beds in total, which have 80 ICU beds. The outbreak occurred in the intensive care unit. The ICU is a third level facility, giving care to surgical, trauma, and immunosuppressed patient populations. 
The infection prevention and control (IPC) team performs active surveillance of hospital-acquired infections (HAI) in all ICUs in the hospital. IPC nurses and doctors work with the ICU and microbiology team when detecting HAls. Centers for Disease Control (CDC) definitions were used for HAI diagnosis. Clinical and active surveillance cultures are checked daily, and the IPC team runs daily rounds in the ICU. IPC runs the outbreak investigation in cooperation with the microbiology department

\section{Outbreak investigation and environmental sampling:}

The outbreak investigation was launched on 11 March 2019 five days after the detection of B. cepacia in respiratory cultures of four different patients. These patients were discussed in the infection control committee. The IPC team performed a field investigation. We defined potential reservoirs and started environmental screening. We sampled the liquid solutions used in patient care, such as chlorhexidine soap, chlorhexidine mouth wash, and ultrasound gel, intubation, ventilation, and oxygenation equipment. We took samples from sink drains in the patient care rooms. Hands of 6 healthcare workers $(\mathrm{HCW})$ were cultured.

Cultures collected from patients and environment, inoculated on plates and incubated at $36^{\circ} \mathrm{C}$ for $18-24$ h. Growth of colonies was detected according to the manufacturer's instructions, and colony species were identified by the VITEK-MS ${ }^{\circledR}$ system (bioMérieux SA, Marcy l'Etoile, France). Antibiotic susceptibility were determined by disk diffusion test and gradient strip test(E-test, bioMérieux).

Pulsed-field gel electrophoresis (PFGE) was done according to the CDC protocol (7). Dendrograms and cluster analysis were generated using the Bionumerics 7.5 (Applied Maths) program with the Unweighted Pair Group Method with the mathematical average (UPGMA) method and the Dice similarity coefficient. Salmonella braenderup ATCC BAA664 isolate was used for molecular size indicator [6].

\section{Results}

Patient 1: A male patient who is 75 years-old with chronic renal disease and myasthenia gravis transferred from a long-term care facility with respiratory failure on 21 February 2019. On day eleven of admission, B. cepacia was isolated from a tracheal aspiration sample. The patient was diagnosed with ventilator-associated pneumonia (VAP) due to B. cepacia, and treatment with piperacillin-tazobactam was started and continued for ten days. The patient improved, but the patient deceased on admission day 55.

Patient 2: A 85 years-old male patient with Alzheimer's was admitted with community-acquired pneumonia to another healthcare facility. Later he was transferred to the ICU. Respiratory samples grew B. cepacia on day 21 of admission. The patient did not fulfill the VAP criteria. On admission day 80 , the patient developed VAP due to Acinetobacter baumannii and died on day 95.

Patient 3: A 86 years-old female patient with sigmoid adenocarcinoma underwent re-laparotomy due to an anastomose leak. The patient was diagnosed with intraabdominal sepsis and treated with piperacillin- 
tazobactam and vancomycin. On admission day two, B. cepacia was detected in the tracheal aspirate. Antimicrobial treatment for intraabdominal sepsis was streamlined to piperacillin-tazobactam and levofloxacin. The patient recovered from intraabdominal infection and was discharged to the surgical ward on admission day 23 and eventually discharged from the hospital on day 25.

Patient 4: A male patient who is 69 years-old with diabetes was admitted with subacute anterior myocardial infarction to the intensive care unit. He underwent left anterior descendent artery stent placement. The patient was intubated due to respiratory insufficiency. On admission day eight, B. cepacia was isolated from respiratory cultures, and the patient was diagnosed with VAP. Cefepime was started then de-escalated to levofloxacin. He received 14 days of antimicrobial treatment. The patient died on admission day 45 .

Patient 5: A male patient who is 73 years-old with chronic obstructive pulmonary disease and chronic renal failure was admitted to the ICU on 24 January 2019. The patient was diagnosed with communityacquired pneumonia and treated with ceftriaxone and clindamycin. B. cepacia was isolated from tracheal aspirate on admission day 60. Levofloxacin treatment was started and continued for ten days. The patient was deceased on admission day 130 .

Patient 6: A male patient who is 77 years-old, operated on 21 January 2019 for cervical spinal stenosis. The patient was admitted to ICU with septic shock on 05 February 2019. On day 56 of admission to ICU, B. cepacia was isolated from deep tracheal aspirate culture. The patient died on the same day. Retrospective examination showed death was not related to a HAl due to B. cepacia.

The six patients' timeline between admission, detection of B. cepacia, and discharge/death is displayed in Figure-1.

In total, we took 34 environmental samples and six hand cultures from HCW. B. cepacia was detected in opened mouthwash products in affected and unaffected patients in the unit. Additional samples were taken from unopened products in the ICU and central storage units. B. cepacia was detected in unopened products. Contamination was detected in all samples (17/17) of a specific batch (G05) of the mouthwash solution. In total, $17 / 20$ of opened and unopened products showed growth. Three unopened solutions without growth had a different batch number (G11). The G05 batch was in use on 24 February 2019 (during the preceding two weeks). The remaining 14 environmental cultures showed no growth other than Pseudomonas aeruginosa and Serratia marcenses from sinks. Cultures from hands of the HCW showed no significant growth.

Overall, six patients became colonized, and three of them developed VAP. The median age of colonized patients was 76 (25-75\%: 73.5-86). The median time to colonization from admission to ICU was 17.5 days (25-75\%: 7.7.5-48.25).

Figure 2 shows the PFGE dendrograms of B. cepacia isolates from the patients and the mouthwash solutions. The PFGE detected five pulsotypes out of $20 \mathrm{~B}$. cepacia isolates. There was a $90 \%$ similarity 
between the two clusters. The isolates in the second cluster had a \%96 similarity.

\section{Interventions and measures taken:}

We contacted the neighboring hospitals using the same product. They reported no additional B. cepacia cases. We recommended to cohort all colonized patients in the same section of the unit. The use of the product was stopped throughout the institution. IPC monitored the hand hygiene compliance of the unit and gave feedback to HCWs during the outbreak. Infection prevention strategies to prevent HAls was reminded to the staff. The outbreak lasted 29 days, and no further clusters were identified after discontinuing the contaminated solution.

\section{Discussion}

The investigation revealed a batch of unopened $2 \%$ CHG mouthwash solution was the source of this B. cepacia outbreak. The PFGE revealed the same strain ( $90 \%$ similarity) that caused the outbreak. The infection control committee took corrective action by the hospital-wide withdrawal of the product. Since the discontinuation of the contaminated solution, we did not detect additional B. cepacia infection or colonization.

Information regarding the role of B. cepacia in healthcare-associated infections in Turkey is scarce. Dizbay et al. reported B. cepacia infection incidence as 0.26 per 1000 admissions, which accounted for $0.7 \%$ of all nosocomial isolates. The most common type of infection was pneumonia. The crude mortality rate of patients with B. cepacia complex was $53.8 \%$ [7].

B. cepacia is clinically relevant in patients with structural lung disease and immunosuppressive patients. If colonized, these patients may develop challenging to treat infections, mostly pulmonary infection.

Given its nature of broad antimicrobial and antiseptic resistance, it can survive in medical solutions [8]. It can also cause outbreaks in non-immunocompromised patients due to contaminated medical equipment and solutions $[4,9]$. Peterson et al. investigated a clonal outbreak of B. cepacia pneumonia in patients without cystic fibrosis. They identified the sink as the source which might have contaminated the respiratory care items [10]. Several studies have reported contamination during manufacturing and after opening the product $[2,11-14]$. Shaban et al. reported a nationwide outbreak of B. cepacia bacteremia in 2017. They isolated the 11 isolates of B. cepacia in 4 hospitals and identified the point source as the contaminated gel packs in sachets used in the sterile ultrasound probe covers [15]. A recent outbreak report showed that contaminated analgesic gel used in urological procedures caused B. cepacia bacteremia in nine patients [16].

This outbreak emphasizes the potential consequences of B. cepacia in critical patients, particularly in intensive care units. Prompt and in-depth epidemiological investigation of such clusters is significant for identifying the source of and controlling the outbreak.

\section{Abbreviations}


CHG: Chlorhexidine

ICU: Intensive care unit

HAl: Hospital-acquired infections

IPC: Infection prevention and control

CDC: Centers for Disease Control

HCW: Healthcare worker

PFGE: Pulsed-field gel electrophoresis

UPGMA: Unweighted Pair Group Method with the mathematical average

VAP: Ventilator associated pneumonia

B. cepacia: Burkholderia cepacia

\section{Declarations}

\section{Ethics:}

The study was conducted according to ethical guidelines approved by the Marmara University School of Medicine. There were no experiments on human participants. The personal data was anonymized and in compliance with local data protection policy.

\section{Consent for publication:}

Not applicable

\section{Availability of data and materials:}

The data is available upon request.

\section{Conflict of interest:}

None

\section{Funding:}

None

\section{Author contributions:}


$\mathrm{HB}, \mathrm{GAG}, \mathrm{FG}, \mathrm{NP}, \mathrm{GC}$, SS, FB, and VK writing and editing the manuscript. HB, FG, and VK treatment of patients. HB, GAG, ES, NP, SS, FB, and GC: outbreak investigation

\section{Acknowledgments:}

None

\section{References}

1. Mahenthiralingam E, Urban TA, Goldberg JB. The multifarious, multireplicon Burkholderia cepacia complex. Nature Reviews Microbiology. 2005;3:144.

2. Ahn Y, Kim JM, Lee Y-J, LiPuma J, Hussong D, Marasa B, et al. Effects of extended storage of chlorhexidine gluconate and benzalkonium chloride solutions on the viability of Burkholderia cenocepacia. J Microbiol Biotechnol. 2017;27:2211-20.

3. Becker SL, Berger FK, Feldner SK, Karliova I, Haber M, Mellmann A, et al. Outbreak of Burkholderia cepacia complex infections associated with contaminated octenidine mouthwash solution, Germany, August to September 2018. Eurosurveillance. 2018;23.

4. Mangram A, Jarvis WR. Nosocomial Burkholderia cepacia outbreaks and pseudo-outbreaks. Infection Control \& Hospital Epidemiology. 1996;17:718-20.

5. Reboli AC, Koshinski R, Arias K, Marks-Austin K, Stieritz D, Stull TL. An outbreak of Burkholderia cepacia lower respiratory tract infection associated with contaminated albuterol nebulization solution. Infection Control \& Hospital Epidemiology. 1996;17:741-3.

6. Tenover FC, Arbeit RD, Goering RV, Mickelsen PA, Murray BE, Persing DH, et al. Interpreting chromosomal DNA restriction patterns produced by pulsed-field gel electrophoresis: criteria for bacterial strain typing. Journal of clinical microbiology. 1995;33:2233.

7. Dizbay M, Tunccan OG, Sezer BE, Aktas F, Arman D. Nosocomial Burkholderia cepacia infections in a Turkish university hospital: a five-year surveillance. The Journal of Infection in Developing Countries. 2009;3:273-7.

8. Tapouk FA, Nabizadeh R, Mirzaei N, Jazani NH, Yousefi M, Hasanloei MAV. Comparative efficacy of hospital disinfectants against nosocomial infection pathogens. Antimicrobial Resistance \& Infection Control. BioMed Central; 2020;9:1-7.

9. Vonberg R-P, Gastmeier P. Hospital-acquired infections related to contaminated substances. Journal of Hospital Infection. 2007;65:15-23.

10. Peterson AE, Chitnis AS, Xiang N, Scaletta JM, Geist R, Schwartz J, et al. Clonally related Burkholderia contaminans among ventilated patients without cystic fibrosis. American journal of infection control. 2013;41:1298-300.

11. Dolan SA, Dowell E, LiPuma JJ, Valdez S, Chan K, James JF. An outbreak of Burkholderia cepacia complex associated with intrinsically contaminated nasal spray. Infection Control \& Hospital Epidemiology. 2011;32:804-10. 
12. Jacobson M, Wray R, Kovach D, Henry D, Speert D, Matlow A. Sustained endemicity of Burkholderia cepacia complex in a pediatric institution, associated with contaminated ultrasound gel. Infection Control \& Hospital Epidemiology. 2006;27:362-6.

13. Gravel-Tropper D, Sample ML, Oxley C, Toye B, Woods DE, Garber GE. Three-year outbreak of pseudobacteremia with Burkholderia cepacia traced to a contaminated blood gas analyzer. Infection Control \& Hospital Epidemiology. 1996;17:737-40.

14. van Laer F, Raes D, Vandamme P, Lammens C, Sion JP, Vrints C, et al. An outbreak of Burkholderia cepacia with septicemia on a cardiology ward. Infection Control \& Hospital Epidemiology. 1998;19:112-3.

15. Shaban RZ, Maloney S, Gerrard J, Collignon P, Macbeth D, Cruickshank M, et al. Outbreak of health care-associated Burkholderia cenocepacia bacteremia and infection attributed to contaminated sterile gel used for central line insertion under ultrasound guidance and other procedures. American journal of infection control. 2017;45:954-8.

16. Zou Q, Li N, Liu J, Li X, Wang Z, Ai X, et al. Investigation of an outbreak of Burkholderia cepacia infection caused by drug contamination in a tertiary hospital in China. American Journal of Infection Control. Elsevier; 2020;48:199-203.

\section{Figures}

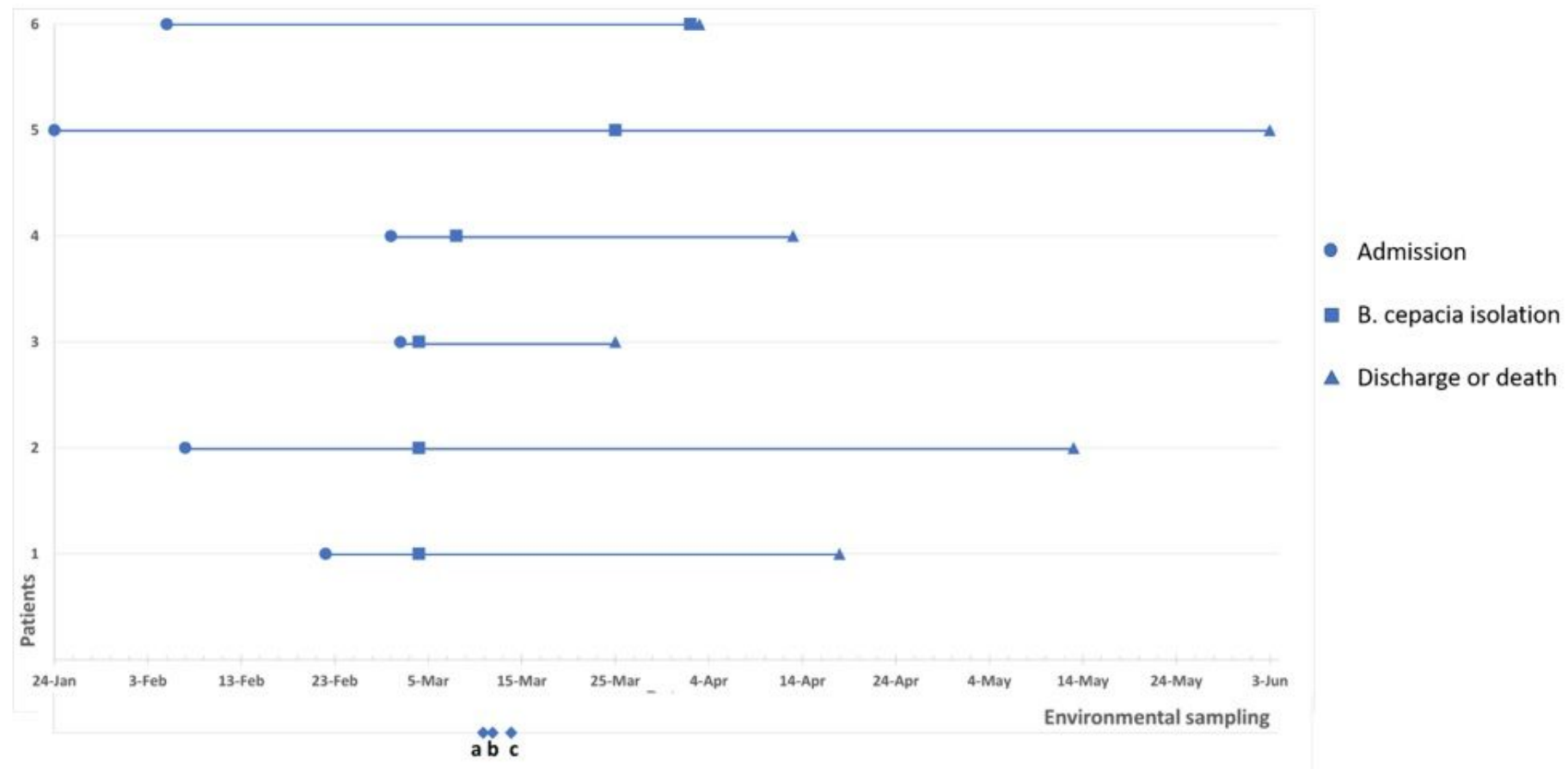

\section{Figure 1}


Timeline of six patients who were admitted to the intensive care unit and had Burkholderia cepacia detected in respiratory specimens during inpatient treatment. Environmental sampling: (a) mouthwash solution of colonized patients and remaining environmental samples, (b) mouthwash solutions of all patients in the unit and unopened stock solutions in the unit, (c) different batches of unopened mouthwash solutions in the storage area.

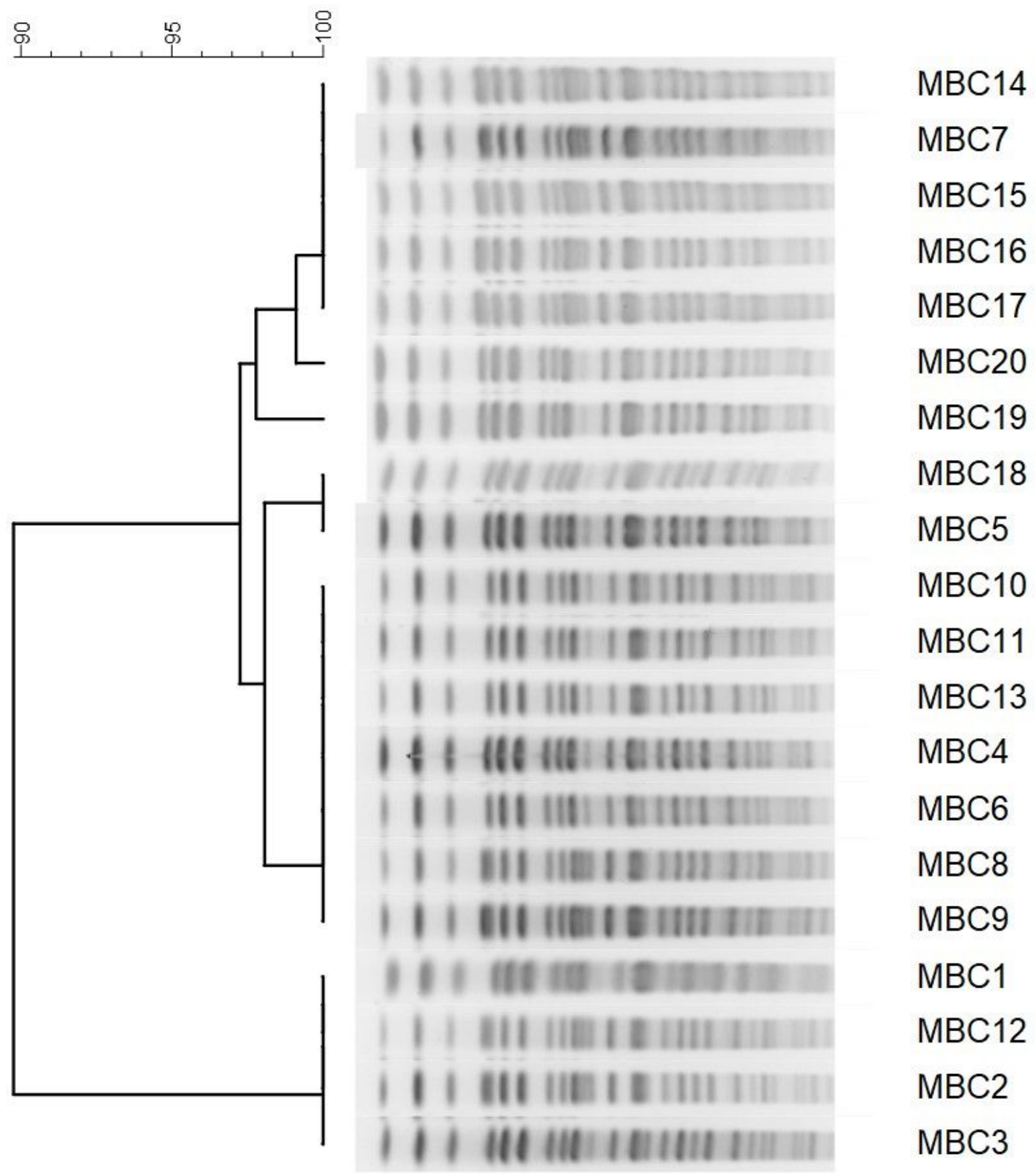

Figure 2 
Pulsed-field gel electrophoresis profiles Spel-digested chromosomal DNA and dendograms from computer-assisted analysis of the profiles. MBC8, 11, 14, 15, 17, and 20 are isolated from the patients' respiratory samples, and the remaining isolates are from mouthwash solutions.

\section{Supplementary Files}

This is a list of supplementary files associated with this preprint. Click to download.

- Graphicalabstract3.png 